\title{
Impact of chronic catheterization and automated blood sampling (Accusampler) on serum corticosterone and fecal immunoreactive corticosterone metabolites and immunoglobulin $\mathrm{A}$ in male rats
}

\author{
F Royo, N Björk, H-E Carlsson, S Mayo and J Hau \\ Division of Comparative Medicine, Department of Neuroscience, Uppsala University, BMC Box 572, 75123 Uppsala, Sweden \\ (Requests for offprints should be addressed to J Hau; Email: jann.hau@bmc.uu.se)
}

\begin{abstract}
Jugular catheters were inserted in nine male rats under general isofluorane anesthesia and the catheters were connected to a commercially available computerized blood sampling device (Accusampler). Blood samples $(150 \mu \mathrm{l})$ were collected every $4 \mathrm{~h}$ during the first $24 \mathrm{~h}$ after surgery and every $12 \mathrm{~h}$ during the following $72 \mathrm{~h}$ until $94 \mathrm{~h}$ after surgery, when the animals were killed. All fecal pellets were collected at blood sampling. Serum corticosterone and fecal concentrations of immunoreactive corticosterone metabolites and immunoglobulin A ( $\operatorname{IgA})$ were quantified by ELISAs. In blood, high corticosterone concentrations $(>200 \mathrm{ng} / \mathrm{ml}$ ) were recorded in the first samples obtained after surgery, but the concentrations decreased steadily during the day and became cyclical, showing a diurnal variation with high levels during evenings and low levels in the mornings. The automatic blood sampling itself did not result in recordable increases in serum corticosterone concentrations. The time delay between the presence of elevated corticosterone levels in blood and in feces was
\end{abstract}

approximately $12 \mathrm{~h}$. Fecal immunoreactive corticosterone metabolite levels remained elevated during the $94 \mathrm{~h}$ study period after surgery. The fecal concentrations of IgA showed substantial between-animal variation and decreased non-significantly after the surgery. Like serum corticosterone, fecal IgA showed a diurnal variation in amounts excreted, in this case with high values in the morning and low values in the evening. The concentrations of fecal corticosterone and IgA were negatively correlated in samples obtained before surgery but no correlation existed after surgery. This indicates that fecal immunoreactive corticosterone metabolites, but not IgA, constitute a good marker of acute stress. For immunoreactive corticosterone metabolites as well as for $\operatorname{IgA}$, the concentration in feces correlated well with total excretion, making single fecal samplings usable as a measure of total secretion.

Journal of Endocrinology (2004) 180, 145-153

\section{Introduction}

In spite of the fact that the severity of an increasing number of human and animal diseases are found to be related to stress, the use and interpretation of stress markers is still a weak point in the study of stress events and their influence in animal or human pathology. From an ethical perspective, there is a moral mandate to strive to reduce stress in laboratory animals during normal husbandry as well as during and after experimental procedures. From a scientific point of view, stress is a well-known and unwanted source of experimental error, because the natural response of an animal to stressors includes alterations in the normal physiology and metabolism. This may add between-animal variation in responses to experimental procedures (Hau et al. 2001, Morton \& Hau 2002). Stress of longer duration induces immunosuppression and increased susceptibility to infectious diseases (Glaser et al. 1987, Klein et al. 1992). This suppression is mediated by adrenal glucocorticoid steroids (Whitten et al. 1998), of which probably the main biologically active hormone in rats is corticosterone (Woodman 1997). Corticosterone increases rapidly in concentration in blood after stressful events and can be used as an index of adrenal function (O'Brien et al. 1995). To reduce stress and release of stress-related hormones, it is desirable to refine experimental procedures, avoiding the stress caused by handling, restraint and sampling. The present paper describes the use of an automatic blood sampling device making it possible to obtain consecutive blood samples from a conscious rat without human interference, once a jugular catheter has been installed. The equipment (Accusampler; Data Innovation Laboratories, Lund, Sweden) is a computerized fully automatic blood sampler, which can be used to deliver drugs into the blood stream as well as to draw blood samples (see http://www.dilab.se). To connect the animal to the device, it is necessary to perform minor surgery under 
Table 1 Changes in the body weight (g) during the trial in each individual rat

\begin{tabular}{|c|c|c|c|c|c|c|c|c|c|}
\hline & \multicolumn{9}{|c|}{ Rat number } \\
\hline & 1 & 2 & 3 & 4 & 5 & 6 & 7 & 8 & 9 \\
\hline & 350 & 356 & 370 & 399 & 499 & 460 & 460 & 510 & 430 \\
\hline Postsurgery & 348 & 350 & 370 & 390 & 490 & 440 & 360 & 520 & 410 \\
\hline
\end{tabular}

general anesthesia, during which a catheter is placed in the jugular vein and/or carotid artery. In spite of this invasive method, the purpose of using this equipment is to make the sampling procedure itself less stressful and more accurate than manual blood sampling in rats.

Non-invasive measures of animal stress may be obtained through quantification of relevant molecules in secretions (e.g. saliva, tears, urine and feces). Fecal corticosteroids have been suggested useful in non-invasive assessment of preceding stress (4-12 $\mathrm{h}$ prior to feces sampling) in a number of species including cats and dogs (Palme et al. 2001, Schatz \& Palme 2001), mice, deer mice and voles (Harper \& Austad 2000, 2001, Touma et al. 2003), rats (Bamberg et al. 2001), roe deer (Dehnhard et al. 2001), and non-human primates (Whitten et al. 1998, Bahr et al. 2000).

The excretion of corticosterone and immunoglobulin A $(\operatorname{IgA})$ in feces has been suggested as an index of stress in rats (Guhad \& Hau 1996, Hau et al. 2001, Pihl \& Hau 2003), and in the present study we analyzed the stressful impact on rats associated with anesthesia and surgery in connection with the use of the Accusampler. The study addressed three main questions: (i) What is the serum corticosterone response in rats following anesthesia and surgical insertion of the catheter and connection to the Accusampler? (ii) What is the relationship between serum corticosterone levels and the amount of immunoreactive corticosterone metabolites secreted with the feces? (iii) What is the relationship between the corticosterone levels in the rat and the amount of IgA secreted with the feces?

\section{Materials and Methods}

\section{Animals}

Nine male outbred Sprague-Dawley rats (B \& K, Sollentuna, Sweden) were used in this study. The animals were housed individually in Macrolone Type III cages in the same room during the study, and their age varied from 8 to 12 weeks, with a weight between 350 and $500 \mathrm{~g}$ (Table 1). The reason for choosing only males in this experiment was to reduce the number of animals used by avoiding the variation attributed to sex (Pihl \& Hau 2003). The rats were kept in standard animal rooms and subjected to standard animal house conditions: light regime $12 \mathrm{~h}$ light:12 h darkness; temperature $21 \pm 1{ }^{\circ} \mathrm{C}$; relative humidity 30-60\%; cages cleaned twice a week. Wooden chips (Finn Tapvei, Finland) were used as bedding material. Water and standard pelleted diet (R36; Laktamin, Stockholm, Sweden) were freely available.

\section{Blood sampling}

Blood samples were collected using the Accusampler, according to manufacturer's recommendations. A catheter was placed in the jugular vein, using the following protocol: the animals were anesthetized with isofluorane (Fluorene; Abbot, Solna, Sweden). The chest and the central part of the neck were shaved and the jugular vein was exposed through an incision approximately $1 \mathrm{~cm}$ above the larynx. A catheter with heparinized saline was introduced into the vein and pushed toward the heart. The catheter was fastened in the vein with two sutures after which it was led s.c. to a Dacron button attached to the skin in the dorsal region of the neck as described in the manufacturer's manual. A common analgesic, buprenorphine (Subutex; Schering-Plough Europe, Belgium) was dissolved in Nutella chocolate, and it was provided at a dose of $0.4 \mathrm{mg} / \mathrm{kg}$ body weight (BW) every day, as a means of postoperative pain relief, as described by Flecknell et al. (1998).

All surgical procedures were performed at $1000 \mathrm{~h}$, and the first blood sample was obtained $2 \mathrm{~h}$ later, at $1200 \mathrm{~h}$. The volume of each blood sample was $150 \mu \mathrm{l}$. Blood samples were collected every $4 \mathrm{~h}$ during the first $24 \mathrm{~h}$ after surgery and every $12 \mathrm{~h}$ during the following $72 \mathrm{~h}$ until $94 \mathrm{~h}$ after surgery when the animals were killed by injecting $100 \mathrm{mg} / \mathrm{kg} \mathrm{BW}$ pentobarbitone (Pentobarbital; Apoteket, Sweden) into the blood stream through the catheter.

\section{Fecal pellet sampling}

Fecal pellets were collected twice daily after the surgery, at 2000 and $0800 \mathrm{~h}$, throughout the experiment. To determine the initial basal levels, fecal pellets were also collected prior to surgery, during 2 days. After collection the pellets were stored at $-20{ }^{\circ} \mathrm{C}$ until analyzed. Prior to extraction of corticosteroids and $\operatorname{IgA}$ the pellets were thawed out on paper tissue, dried in a heat cabinet at $30{ }^{\circ} \mathrm{C}$ for $2 \mathrm{~h}$ and weighed.

\section{Analysis of $I g A$ and corticosterone}

The corticosterone concentration in serum was analyzed without any preceding extraction procedures. The analysis of fecal molecules, however, was performed following the extraction procedures for corticosteroids and $\operatorname{IgA}$ from feces described previously (Hau et al. 2001, Pihl \& Hau 2003). IgA was quantified using the ELISA technique and reagents described in Hau et al. (2001). The intra-assay 


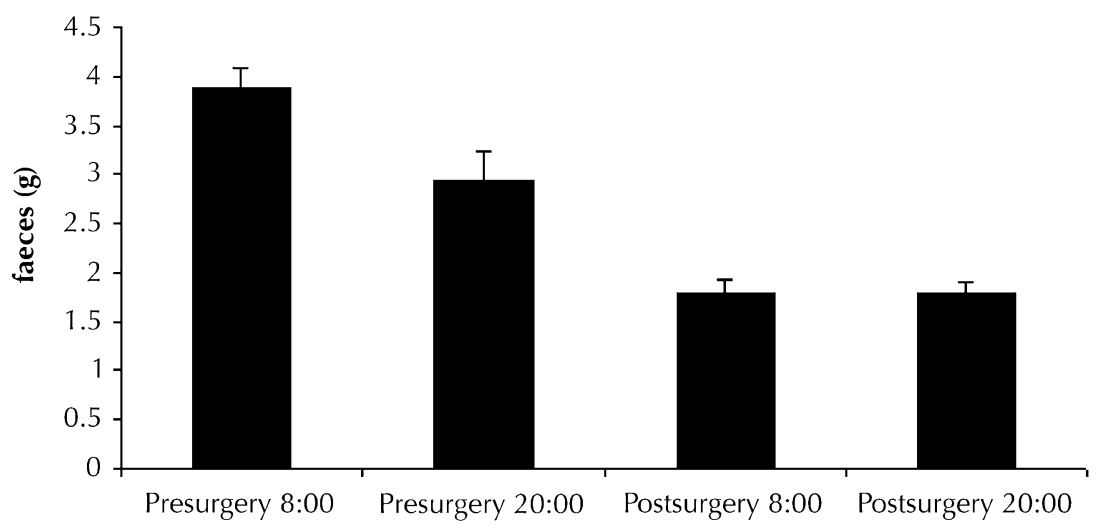

Figure 1 Mean fecal weight presented as average of each period during morning and evening. The error bars represent S.E.M. $(n=9)$.

coefficient of variation was $2 \cdot 3 \%$ and the inter-assay coefficient $5 \cdot 8 \%$. Immunoreactive corticosterone metabolites were quantified using an OCTIGEN ELISA kit (Immuno-Diagnostic Systems Ltd; Tyne \& Wear, UK) according to the manufacturer's instructions. The crossreactivity of this kit is $<0.05 \%$ of reactivity against deoxycorticosterone, cortisol and 5- $\alpha$-pregranedione and $<0.01 \%$ for cortisone, 17 - $\alpha$-hydroxypregnenolone, tetrahydrocortisone, 11-dehydro-corticosterone, deoxycortisol, 11-desoxycortisol and 4-pregnan-20-alpha-ol-3one. The intra-assay coefficient of variation was $2 \cdot 1 \%$ and the inter-assay coefficient $6 \cdot 3 \%$.

The stability of immunoreactive corticosterone metabolites and IgA in fresh rat feces was analyzed by quantification of these molecules in a pool $(n=4)$ of feces which was mixed, aliquoted and frozen after having been stored at room temperature for various time spans: 1, 2, 4, 6, 12 and $24 \mathrm{~h}$. The concentration of the respective molecules did not differ significantly $(<10 \%$ variation in concentration between the samples) regardless of the time the samples had been left at room temperature prior to freezing.

\section{Statistics}

The statistical analysis was performed according to Svendsen \& Hau (1981). ANOVA analysis was performed using Microcal Software Inc., Northampton, MA, USA and correlation coefficients and trend lines were established using Microsoft Excel (Microsoft Corporation). $P<0.05$ were considered significant.

\section{Ethics committee approval}

The experimental procedures were approved by the Uppsala Regional Ethics Committee in Tierp, Sweden.

\section{Results}

Effect of experimental procedure on $B W$ and amount of feces excreted

The majority of the rats lost weight during the experiment (Table 1). The amount of feces excreted in the initial period after surgery was significantly smaller that the amount secreted prior to surgery per time unit (Fig. 1).

\section{Corticosterone concentration in serum and amount excreted in} feces

The serum corticosterone concentrations were high $(>200 \mathrm{ng} / \mathrm{ml})$ in the first two samples after surgery ( 2 and $6 \mathrm{~h})$ after which a cyclicity in concentration fluctuations was observed with peaks of approximately $100 \mathrm{ng} / \mathrm{ml}$ every $12 \mathrm{~h}$ (evenings) as shown in Fig. 2A. The amounts of fecal immunoreactive corticosterone metabolites excreted in feces were significantly increased after surgery (Fig. 2B) when all mean values measured before and after surgery were compared.

\section{Excretion profile of $\operatorname{Ig} A$}

The profile of IgA secretion, expressed as $\mu \mathrm{g} \operatorname{IgA} / \mathrm{h}$ and $/ \mathrm{kg} \mathrm{BW}$ is presented in Fig. 3. Although the mean values of IgA excreted after surgery were generally lower than those recorded before surgery, this difference was not significant. After surgery the IgA amounts excreted varied with a diurnal rhythm with peaks every $12 \mathrm{~h}$ (mornings).

Correlation between excreted amounts of corticosterone and $\operatorname{Ig} A$

A significant negative correlation $(r=-0.53, n=17$, $P<0 \cdot 05)$ between the mean values of fecal immunoreactive corticosterone metabolites excreted in the 


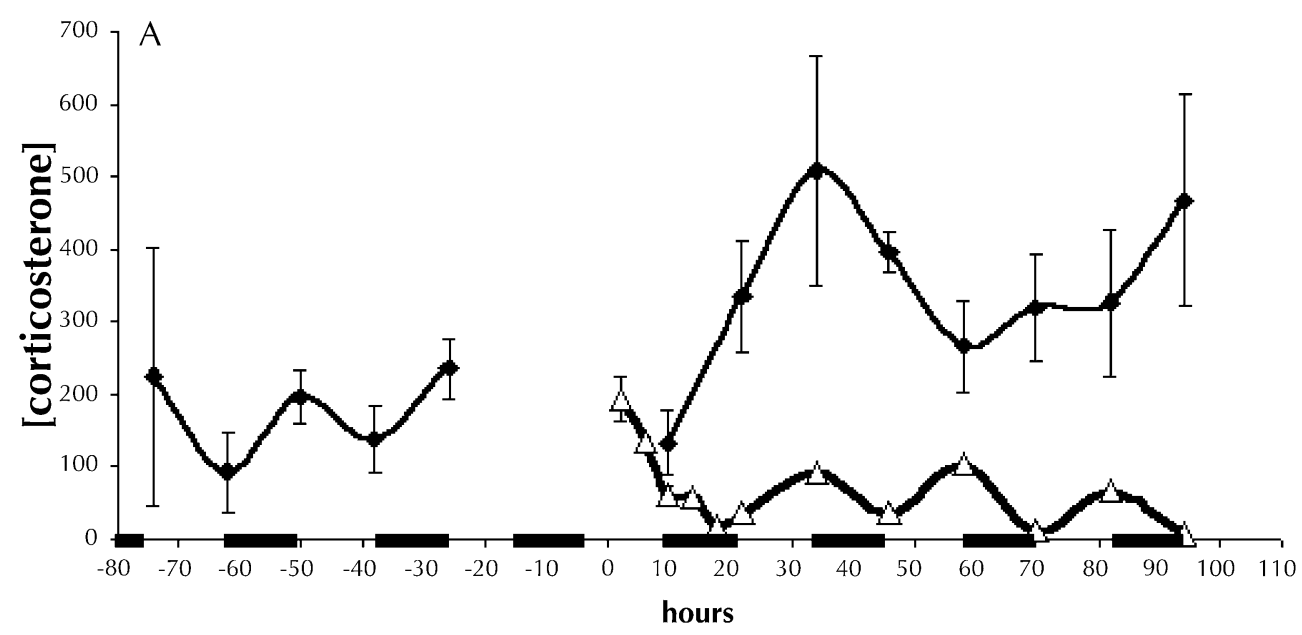

$\rightarrow$ Faeces (ng/h kg bw) $\rightarrow-\operatorname{Serum}(\mathrm{ng} / \mathrm{ml})$

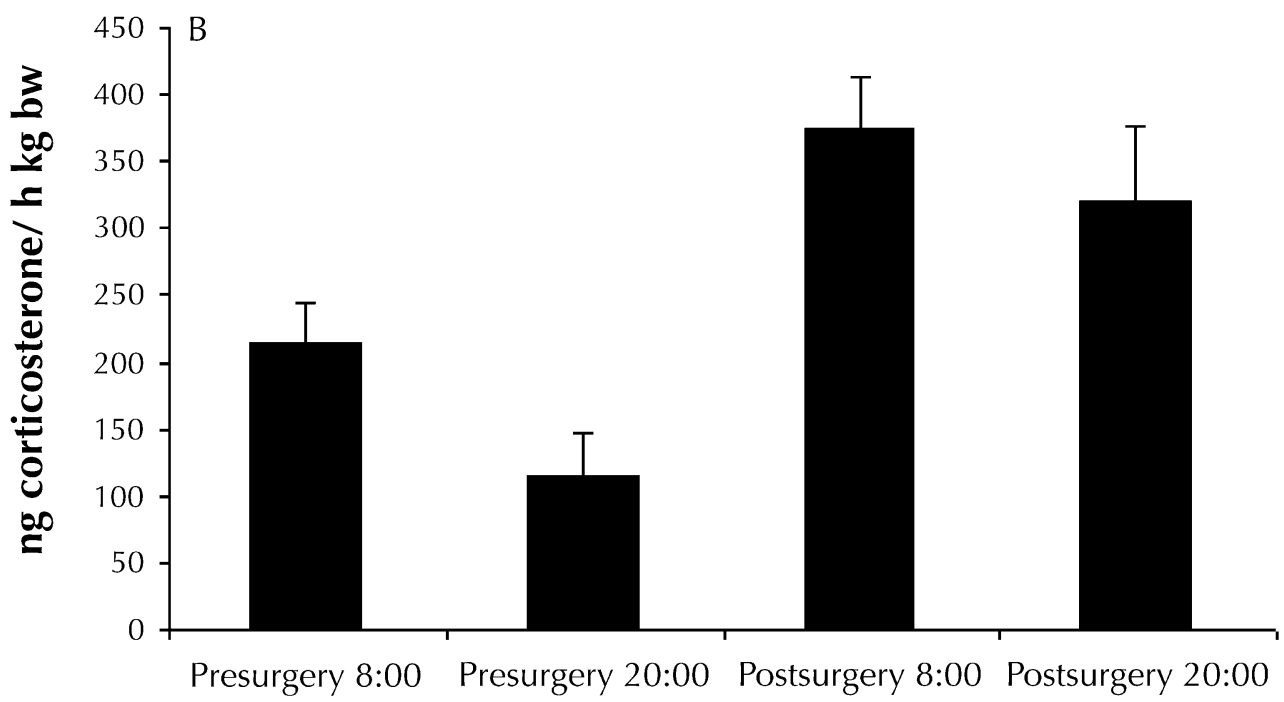

Figure 2 (A) Corticosterone serum levels and excretion profile in feces of immunoreactive corticosterone metabolites. The point 0 represents the surgery time $(1000 \mathrm{~h})$. Therefore, all the samples represented in a time point were collected at the same hour of the day, i.e. 2000 or 0800 h. (B) Average of total excretion of immunoreactive corticosterone metabolites in feces collected at 0800 and $2000 \mathrm{~h}$ before and after surgery. In both cases the dark bars in the $x$ axis represent dark periods (from 2000 to $0800 \mathrm{~h}$ ) and the error bars \pm S.E.M. $(n=9)$.

mornings and evenings and the mean values of $\operatorname{IgA}$ excreted in the evenings and in the mornings was found in samples obtained prior to surgery (Fig. 4A). However, in the samples obtained after surgery there was no significant correlation $(r=-0 \cdot 19, n=17, P>0 \cdot 05)$ between the secreted amounts of the molecules (Fig. 4B).

\section{Relationship between concentration in feces and total amount of} excreted IgA and corticosterone

There was a positive correlation between the concentration of $\operatorname{IgA}$ and immunoreactive corticosterone metabolites in the fecal pellets, and the total amount of both molecules secreted per hour and $\mathrm{kg} \mathrm{BW}$ during the study periods. The IgA correlation value was $r=0.72$ $(n=99, P<0 \cdot 001)$ and for corticosterone $r=0.89(n=96$, $P<0 \cdot 001)$ (Fig. 5).

\section{Discussion}

The automated blood sampling device used in the present study (Accusampler) has been developed to allow blood sampling and administration of substances without 


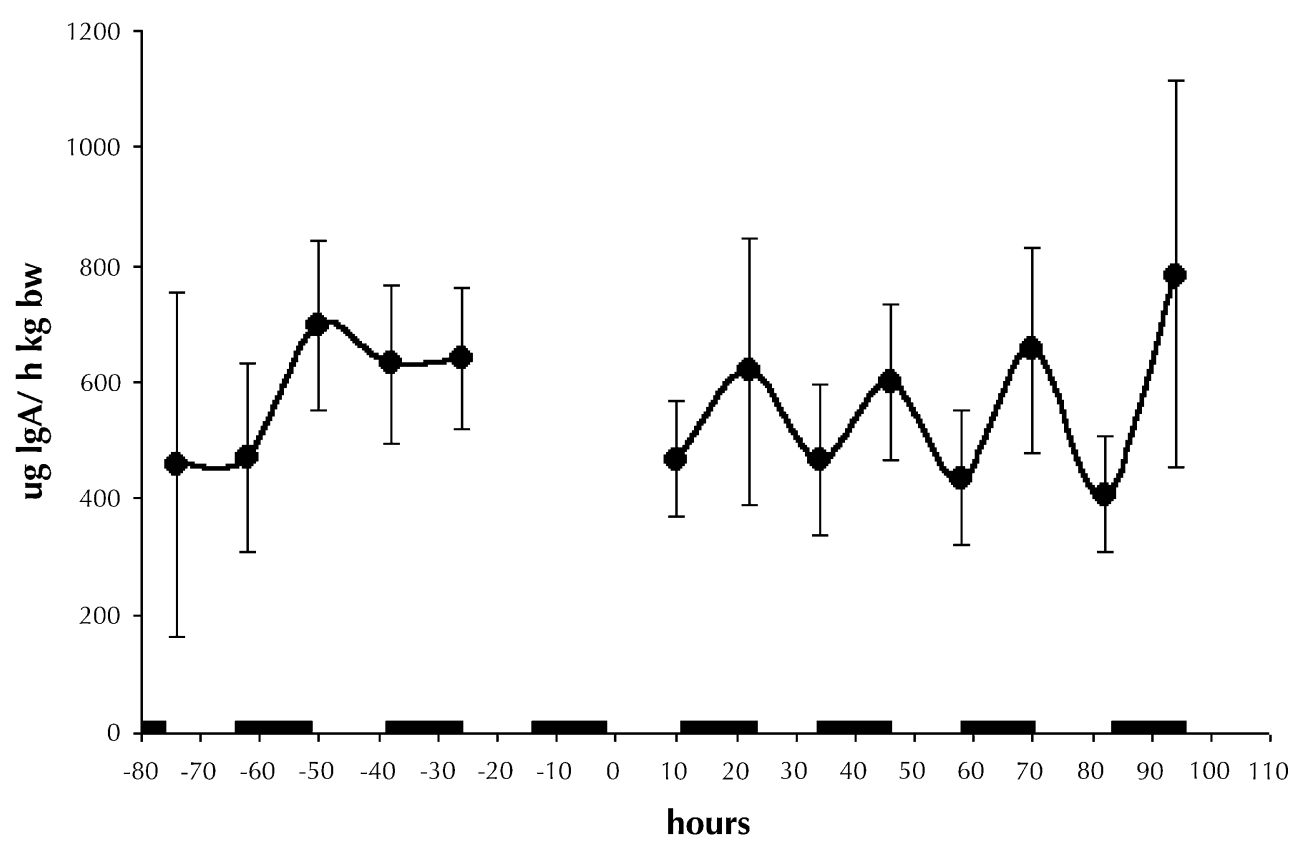

Figure $3 \mathrm{IgA}$ excretion profiles. As in Fig. 2A, the point 0 represents the time of surgery, the dark bars in the $x$ axis represents dark periods (from 2000 to 0800 h) and the error bars \pm S.E.M. $(n=9)$.

disturbance to the animals. However, in order to implant a catheter in the animal it is necessary to perform an invasive procedure which requires surgical anesthesia, which is a well-known stress factor (O'Brien et al. 1995, Whitten et al. 1998). Consequently, an initial negative impact on the animal's well-being is to be expected after surgery. A transient reduction of food intake and BW loss as described for humans (Jemmott \& Magloire 1988) was thus to be expected in the rats of the present study. Considering that the rats employed in our study were young and growing, the effect on BW gain was obvious. One of the rats increased slightly in BW during the trial, but five rats lost between 2 and 3\% and rat number $7>20 \%$ of BW. We did not measure the consumption of food because it was distributed on the cage floor to facilitate access and thus became mixed with the bedding making accurate weighing difficult. However, there was a transient decrease in feces production after surgery, which is a good measure of food intake in rodents.

It is well known that stress associated with anesthesia without surgery results in elevated levels of corticosteroids in the circulation (O'Brien et al. 1995) and this also includes isofluorane (Vachon \& Moreau 2001), which was used in the present study. Not unexpectedly we recorded high levels of serum corticosterone in the first samples obtained after surgery. The highest concentrations were measured in the first sample after surgery after which a continuous decrease during the next $24 \mathrm{~h}$ was observed. In accordance with good veterinary practice the rats were provided with an opioid agonist, buprenorphine, as pain relief. This drug may reduce the corticosterone levels in rats (Gomez-Flores \& Weber 2000). Twenty-four hours after surgery, the concentrations began to fluctuate in a daily rhythm with high concentrations in the evening and low concentrations in the morning. A similar pattern has been registered previously by Windle et al. (1998a,b), who found values very similar to the ones reported in the present study, which are also in the same range $(100 \mathrm{ng} / \mathrm{ml})$ as described by Gärtner et al. (1980) in unstressed rats. This rather quick return to normality, even if stress persists, has been previously described by Manser (1992). In the present study we recorded an increase of excretion of fecal immunoreactive corticosterone metabolites after the surgery $(22 \mathrm{~h})$ compared with the basal levels measured before surgery, and this suggests a delay between serum corticosterone changes and excreted corticosterone and immunoreactive corticosterone metabolites, which is in agreement with the findings of Teskey-Gerstl et al. (2000) and Bamberg et al. (2001). Since the OCTIGEN ELISA kit has been developed to measure the corticosterone concentration in serum and not in feces, it is uncertain to what extent the kit will quantify corticosterone metabolites present in feces. Consequently, we have chosen in the present paper to use the term 'immunoreactive corticosterone metabolites' instead of 'corticosterone' when we address fecal concentrations. The nature of these fecal metabolites is not well known, but Touma et al. (2003) found several peaks of radioactivity in chromatograms following blood infusion of $\left[{ }^{3} \mathrm{H}\right]$ corticosterone, which suggested than this hormone is 


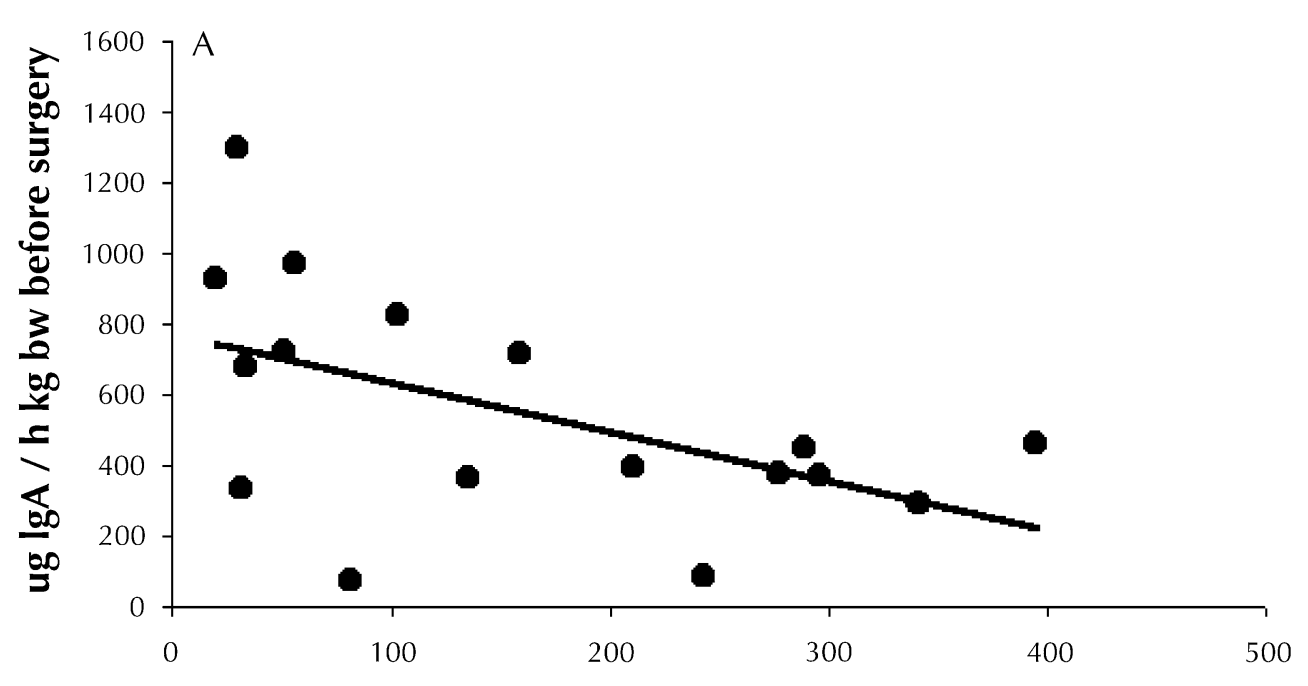

ng corticosterone / $\mathrm{h}$ kg bw before surgery

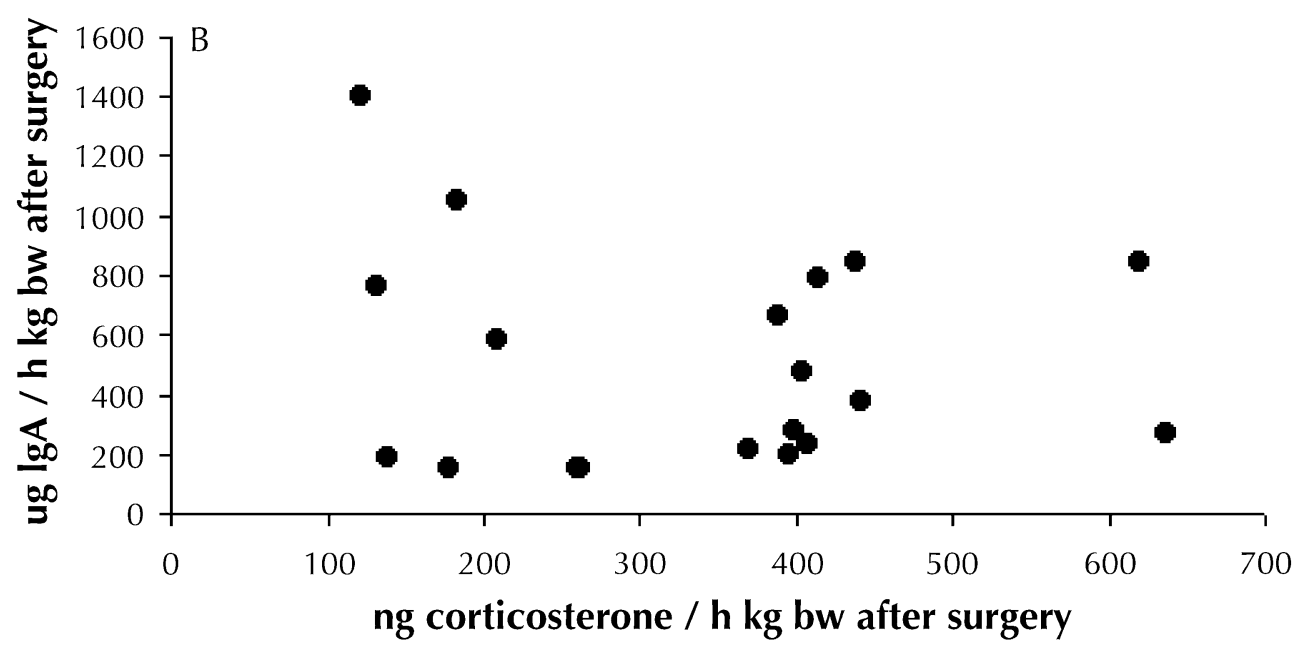

Figure 4 (A) Correlation between the mean values of immunoreactive corticosterone metabolites concentration in feces before surgery and the mean value of $\lg \mathrm{A}$ in the same rat and period. The data have been blocked as mornings and evenings, and the correlation has been done between the corticosterone metabolites concentration values in the morning and the $\lg A$ concentration values in the evening. (B) This correlation disappeared after surgery.

metabolized or degraded into several metabolites. To the best of our knowledge there are no groups who have attempted to establish assays allowing quantification of all peaks. Bamberg et al. (2001) have developed antisera against relevant corticosterone metabolites but using an immunoassay based on an antibody against a corticosterone metabolite, albeit a major one, is not necessarily more biologically relevant than measuring corticosterone itself, perhaps including some cross-reacting metabolites that may react with the antiserum. It is perhaps important to emphasize that we experienced no difficulties measuring generous levels of immunoreactive corticosterone metabolites in rat feces and that the concentrations we recorded are in good agreement with the levels of fecal corticosterone (native molecular form only) measured using HPLC quantification (Guhad et al. 2003).

Before the surgery, a trend to cyclicity in the excretion of immunoreactive corticosterone metabolites was observed. Higher amounts were secreted in the feces collected at $0800 \mathrm{~h}$ than in the feces collected at $2000 \mathrm{~h}$. This result is in agreement with previous studies of male Sprague-Dawley rats (Bamberg et al. 2001, Pihl \& Hau 2003) and this rhythm is opposite to that of serum corticosterone, and confirms the mentioned delay observed after surgery. After the surgery this cyclicity was lost (Fig. 2A), and a similar effect has also been reported in 

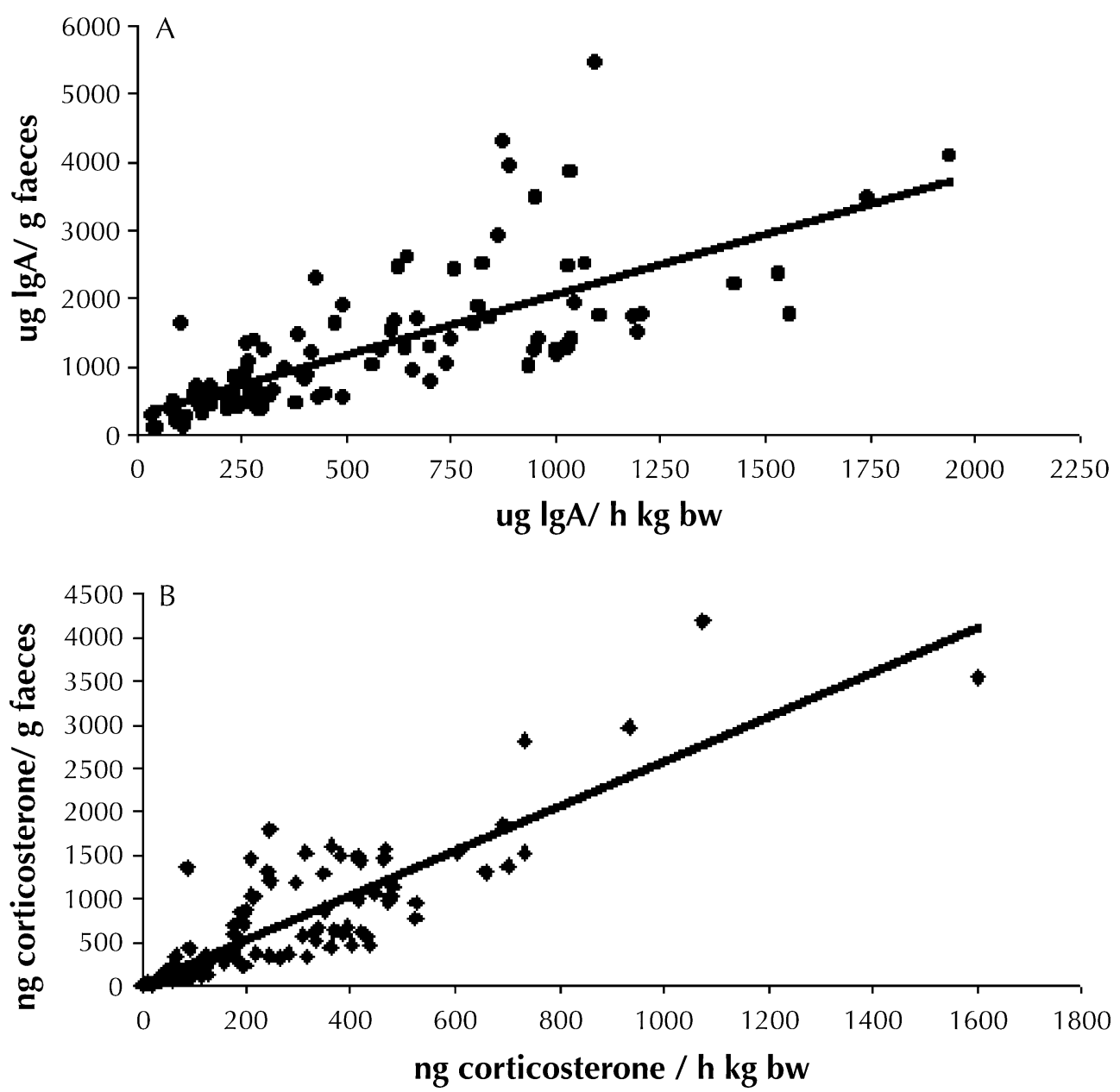

Figure 5 Correlation between the fecal concentration and the total excretion per hour and $\mathrm{kg}$ BW of (A) $\lg \mathrm{A}$ and (B) immunoreactive corticosterone metabolites.

monkeys (Quabbe et al. 1982) and sheep (Przekop et al. 1985) after stressful events.

Windle et al. $(1998 a, b)$ found that serum corticosterone levels are not constant in the rat, because this hormone is released in a pulsatile manner. The duration and intensity of the peaks during the night were higher than during the day. Whitten et al. (1998) suggested that excreted steroids were a better measure of overall stress than serum levels, and if this is indeed the case the consistently higher fecal levels after surgery in our study indicate that the animals were still exhibiting a stress response $94 \mathrm{~h}$ after surgery.

A number of studies have demonstrated that increased serum corticosterone levels are associated with a decrease in secretory IgA (O'Connor \& Corrigan 1987, Skandakumar et al. 1995, Hucklebridge et al. 1998). In the present study, however, the lower amounts of IgA secreted after surgery as compared with the levels before surgery were not significant. This may be due to great variation between the levels of secretory IgA between individual animals. The amount of IgA excreted by the animal with the highest levels was almost 10 times greater than the values of the animal with the lowest levels. This great between-animal variation has not been reported in previous studies (Hau et al. 2001, Pihl \& Hau 2003) and it may be related to the differences in weight or age of the rats. It is interesting that fecal secretions of $\operatorname{IgA}$ exhibit a diurnal rhythm very similar to that of serum corticosterone but with a delay of $12 \mathrm{~h}$. Indeed a significant negative correlation was found between the excreted amounts of corticosterone and $\operatorname{IgA}$ in samples obtained before surgery, which indicates than IgA may be a useful marker for assessment of the well-being of a population, and confirms previous studies where IgA has been proposed as a marker of long-term stress (30-90 days) (Valdimarsdottir \& Stone 1997). However, in the samples obtained after surgery this correlation was not present. This suggests that stressinduced changes in excreted IgA concentrations are slower and perhaps less pronounced than those of corticosteroids. 
Therefore, both stress markers may be useful for assessing animal well-being, IgA for long-term well-being and corticosterone for detecting acute stress events. Interestingly, our results indicate that $\operatorname{IgA}$ concentrations are not markedly influenced by short-term transient stress and this may be useful to distinguish between, for example, distress caused by poor husbandry and acute stress due to handling and experimental procedures.

A good correlation was found between the respective concentrations of IgA and immunoreactive corticosterone metabolites in feces, and the total excreted amounts of these molecules. This confirms the results published by Pihl \& Hau (2003), and this property may prove useful because it indicates that single fecal samples can be used as an easy diagnosis of stress. It may not be necessary to collect all the feces, as the total secretion can be estimated based on the concentration per gram of feces in a single sample.

Manual blood sampling normally results in a stress response and the catheterization of the rats in the present study clearly resulted in an initial stress response. It is interesting, however, that the rats recovered quickly and already exhibited low serum corticosterone levels $18 \mathrm{~h}$ after surgery followed by a cyclical diurnal rhythmicity. This indicates that the results obtained using the Accusampler in pharmacological and physiological studies may not be biased by a strong acute stress response in the animals. This automatic blood-sampling device was able to draw blood samples without inducing a corticosterone release in blood. Although the use of this equipment necessitates an invasive procedure to install the catheter and connect the rat to the autosampler, which produced a stress response characterized by an increase of the total release of corticosterone and an increase of fecal immunoreactive corticosterone metabolites, the blood sampling itself seems to be associated with little or no stress.

\section{Acknowledgements}

We are grateful for the technical assistance of Pär Davidsson, Klas Abelson, Else Marie Andersson and Urban Höglund.

\section{Funding}

F R was supported by a grant from Diputación General de Aragón, Formacion de Personal Investigador, ref. B004/2000. Data Innovation Laboratories, Lund, Sweden generously donated two Accusamplers.

\section{References}

Bahr NI, Palme R, Mohle U, Hodges JK \& Heistermann M 2000 Comparative aspects of the metabolism and excretion of cortisol in three individual nonhuman primates. General and Comparative Endocrinology 117 427-438.
Bamberg E, Palme R \& Meingassner JG 2001 Excretion of corticosteroid metabolites in urine and faeces of rats. Laboratory Animals 35 307-314.

Dehnhard M, Clauss M, Lechner-Doll M, Meyer HH \& Palme R 2001 Noninvasive monitoring of adrenocortical activity in roe deer (Capreolus capreolus) by measurement of fecal cortisol metabolites. General and Comparative Endocrinology 123 111-120.

Flecknell PA, Roughan JV \& Stewart R 1998 Use of oral buprenorphine ('buprenorphine jello') for postoperative analgesia in rats - a clinical trial. Laboratory Animals 33 169-174.

Glaser R, Rice J, Sheridan J, Fertel R, Stoot J, Speicher C, Pinsky D, Kotur M, Post A \& Beck M 1987 Stress-related immune suppression: health implications. Brain, Behavior, and Immunity $\mathbf{5}$ $7-20$.

Gomez-Flores R \& Weber RJ 2000 Differential effects of buprenorphine and morphine on immune and neuroendocrine functions following acute administration in the rat mesencephalon periaqueductal gray. Immunopharmacology 48 145-156.

Guhad FA \& Hau J 1996 Salivary IgA as a marker of social stress in rats. Neuroscience Letters 216 1-4.

Guhad FA, Nevalainen T \& Lang CM 2003 Fecal corticosterone: a non invasive method of stress assessment in rats. Contemporary Topics in Laboratory Animal Science 42 72-73 (Abstract PS28).

Gärtner K, Buttner D, Dohler K, Friedel R, Lindena J \& Trautschold I 1980 Stress response of rats to handling and experimental procedures. Laboratory Animals 14 267-274.

Harper JM \& Austad SN 2001 Effect of capture and season on fecal glucocorticoid levels in deer mice (Peromyscus maniculatus) and red-backed voles (Clethrionomys gapperi). General and Comparative Endocrinology 123 337-344.

Harper JM \& Austad SN 2000 Fecal glucocorticoids: a noninvasive method of measuring adrenal activity in wild and captive rodents. Physiological and Biochemical Zoology 73 12-22.

Hau J, Andersson E \& Carlsson H-E 2001 Development and validation of a sensitive ELISA for quantification of secretory IgA in rat saliva and faeces. Laboratory Animals 35 301-306.

Hucklebridge F, Clow A \& Evans P 1998 The relationship between salivary secretory immunoglobulin A and cortisol: neuroendocrine response to awakening and the diurnal cycle. International Journal of Psychophysiology 31 69-76.

Jemmott JB \& Magloire K 1988 Academic stress, social support, and secretory immunoglobulin A. Journal of Personal and Social Psychology 55 803-810.

Klein F, Lemaire V, Sandi C, Vitiello S, Van der Logt J, Laurent PE, Neveu P, Le Moal M, \& Mormede P 1992 Prolonged increase of corticosterone secretion by chronic social stress does not necessarily impair immune functions. Life Sciences 50 723-731.

Manser CE 1992 The Assessment of Stress in Laboratory Animals, pp 29-54. Horsham: RSPCA.

Morton DB \& Hau J 2002 Welfare assessment and humane endpoints. In Handbook of Laboratory Animal Science, vol 1, edn 2, pp 457-486. Eds J Hau \& L Van Hoosier Jr. Boca Raton, FL: CRC Press.

O’Brien D, Tibi Opi P, Stodulski G, Saibaba P \& Hau J 1995 Stress perception of surgical anaesthesia in rats. In Proceedings of the Fifth FELASA Symposium: Welfare and Science, pp 24-27. Ed J Bunyan. London: Royal Society of Medicine Press.

O'Connor PJ \& Corrigan DL 1987 Influence of short-term cycling on salivary cortisol levels. Medicine and Science in Sports and Exercise 19 224-228.

Palme R, Schatz S \& Mostl E 2001 Effect of vaccination on fecal cortisol metabolites in cats and dogs. Deutsche Ttierarztliche Wochenschrift 108 23-25.

Pihl L \& Hau J 2003 Faecal corticosterone and immunoglobulin A in young adult rats. Laboratory Animals 37 166-171.

Przekop F, Stupnicka E, Wolinska-Witort E, Mateusiak K, Sadowski B \& Domanski E 1985 Changes in circadian rhythm and suppression of the plasma cortisol level after prolonged stress in the sheep. Acta Endocrinologica 110 540-545. 
Quabbe HJ, Gregor M, Bumke-Vogt C \& Hardel C 1982 Pattern of plasma cortisol during the 24-hour sleep/wake cycle in the rhesus monkey. Endocrinology 110 1641-1646.

Schatz S \& Palme R 2001 Measurement of faecal cortisol metabolites in cats and dogs: a non-invasive method for evaluating adrenocortical function. Veterinary Research Communications 25 271-287.

Skandakumar S, Studolski G \& Hau J 1995 Salivary IgA as a stress marker in dogs. Animal Welfare 4 339-350.

Svendsen P \& Hau J 1981 Laboratory Animals and Animal Experimentation [in Danish]. Odense: Odense Universitetsforlag.

Teskey-Gerstl A, Bamberg E, Steineck T \& Palme R 2000 Excretion of corticosteroids in urine and faeces of hares (Lepus europaeus). Journal of Comparative Physiology 170 163-168.

Touma C, Sachser N, Mostl E \& Palme R 2003 Effects of sex and time of day on metabolism and excretion of corticosterone in urine and feces of mice. General and Comparative Endocrinology 130 267-278.

Vachon P \& Moreau JP 2001 Serum corticosterone and blood glucose in rats after two jugular vein blood sampling methods: comparison of the stress response. Contemporary Topics in Laboratory Animal Science 40 22-24.

Valdimarsdottir HB \& Stone AA 1997 Psychosocial factors and secretory immunoglobulin A. Critical Reviews in Oral Biology and Medicine 8 461-474.
Whitten PL, Brockman DK \& Stavisky RC 1998 Recent advances in noninvasive techniques to monitor hormone-behavior interactions. Yearbook of Physiological Anthropology 41 1-23.

Windle RJ, Wood SA, Lightman SL \& Ingram CD 1998a The pulsatile characteristics of hypothalamo-pituitary-adrenal activity in female Lewis and Fischer 344 rats and its relationship to differential stress responses. Endocrinology 139 4044-4052.

Windle RJ, Wood SA, Shanks N, Lightman SL \& Ingram CD $1998 b$ Ultradian rhythm of basal corticosterone release in the female rat: dynamic interaction with the response to acute stress. Endocrinology 139 443-450.

Woodman DD 1997 The adrenal glands. In Laboratory Animal Endocrinology: Hormonal Action, Control Mechanisms and Interactions with Drugs, pp 253-286. Chichester: John Wiley \& Sons Ltd.

Received 19 September 2003

Accepted 1 October 2003 